OHSTPY-HEP-T-97-002

February 1997

\title{
Gauge-mediated SUSY Breaking at an Intermediate Scale
}

\author{
Stuart Raby \\ Department of Physics, The Ohio State University, Columbus, Ohio 43210
}

\begin{abstract}
Gauge-mediated SUSY breaking with a messenger scale of order $10^{15} \mathrm{GeV}$ has some interesting features. It can solve the flavor changing neutral current problem of supersymmetric models with predictions for superpartner masses which are identical to those of minimal supergravity models. It can however also lead to theories with novel experimental signatures. For example, we present a model in which the gluino is the lightest supersymmetric particle. We also review the present experimental status of a stable gluino.
\end{abstract}




\section{Introduction}

Supersymmetry [SUSY] breaking in a "hidden" sector can be transmitted to the observable sector through Standard Model [SM] gauge interactions [四, 2] or through supergravity interactions [3]. In supergravity theories, SUSY breaking in the observable sector is set by the gravitino mass with

$$
m_{3 / 2}=\frac{F}{\sqrt{3} M_{\text {Planck }}} .
$$

$F$ is the fundamental scale of SUSY breaking and

$$
M_{\text {Planck }} \equiv 1 / \sqrt{8 \pi G_{N}}=2.4 \times 10^{18} \mathrm{GeV}
$$

is the messenger scale. In gauge mediated SUSY breaking, on the other hand, $M$ is the mass of the messenger states which have the property that they

- couple directly to the SUSY breaking sector, and

- communicate with the observable sector predominantly through SM gauge interactions.円

Low energy messenger models have been discussed recently [4]. In these theories, soft SUSY breaking mass terms, for scalars and gauginos, scale as local dimension 2 and 3 operators, respectively, up to the messenger scale. Above $M$, soft SUSY breaking mass terms no longer scale as local mass operators (see Appendix 1). As a result, low energy messenger models solve the flavor changing neutral current [FCNC] problems of SUSY [5, 6], since

- scalars with the same SM quantum numbers are degenerate, and

- the low energy physics is not sensitive to the physics above $M$.

In addition, these theories can lead to observable low energy effects of SUSY breaking, i.e. a distinct spectrum of squark and slepton masses or new types of SUSY signatures for accelerator experiments[7.

In the minimal messenger scenario 4 , scalars and gauginos get mass of order

$$
\frac{\alpha}{4 \pi} \frac{F_{X}}{M} \sim \frac{\alpha}{4 \pi} M
$$

\footnotetext{
${ }^{1}$ Gravity mediated SUSY breaking is always present.
} 
where $F_{X} \sim M^{2}, M \sim 10-100 \mathrm{TeV}$ and $\alpha$ is the fine structure constant associated with the SM charge carried by the particular scalar or gaugino. In this paper we describe a simple generalization of the minimal messenger scenario which illustrates the following points -

1. The SUSY breaking scale $\sqrt{F_{X}}$ and the messenger scale $M$ are not necessarily the same. The only necessity is that

$$
\Lambda=\frac{F_{X}}{M} \sim 10-100 \mathrm{TeV}
$$

is fixed. In our example we shall show that $M$ can have any value from $\sim 10-100 \mathrm{TeV}$ to $M_{\text {Planck. }}$. Hence the two SUSY breaking schemes - supergravity mediated SUSY breaking (with $M \sim M_{\text {Planck }}$ ) and the minimal gauge mediated SUSY breaking (with $M \sim 10-100 \mathrm{TeV}$ ) are just two extreme limits in a continuum of possible theories.

A particularly interesting case is when $M \approx M_{G U T}[2]$. In this case, SUSY breaking is transmitted to the observable sector through GUT interactions. As a result, squarks and sleptons within a complete GUT multiplet are degenerate, assuming the messenger masses are invariant under the GUT symmetry. Such theories look very much like the minimal supergravity models [3] (or constrained minimal supersymmetric standard model[CMSSM] [8]).

2. In order to generate gaugino masses, it is necessary to break both SUSY and $\mathrm{R}$ symmetry. In the minimal scenario, they are broken at the same scale. We shall give a simple example in which the $\mathrm{R}$ symmetry breaking scale $M_{R}$ is less than the messenger scale; thus suppressing gaugino masses.

3. Finally, even in a GUT it is possible to obtain non-degenerate gaugino masses at $M \approx M_{G U T}$. In our example, gluinos may be the lightest supersymmetric particles; resulting in very interesting experimental consequences. 


\section{Intermediate Messenger Scales}

We illustrate these ideas in a simple SO(10) SUSY GUT.E The superpotential is given by

$$
W=\lambda_{\mathcal{S}} \mathcal{S} 10^{2}+X\left(10^{2}+\lambda \phi^{+} \phi^{-}+\lambda^{\prime} X^{2}\right)
$$

where $\mathcal{S}, X$ are $\mathrm{SO}(10)$ singlets, $\phi^{+}, \phi^{-}$are also $\mathrm{SO}(10)$ singlets with charge \pm 1 under a messenger $\mathrm{U}(1)$ and 10 is a ten under $\mathrm{SO}(10)$. Note that we assume SUSY breaking occurs in a "hidden" sector dynamically and transmits this directly to the states $\phi^{+}, \phi^{-}$via the messenger $U(1)$ resulting in a negative mass squared for these two states 四.

The scalar potential is thus given by

$$
\begin{aligned}
& V=\quad \lambda_{\mathcal{S}}^{2}\left|10^{2}\right|^{2}+\left|10^{2}+\lambda \phi^{+} \phi^{-}+3 \lambda^{\prime} X^{2}\right|^{2} \\
& +\left|\left(\lambda_{\mathcal{S}} \mathcal{S}+X\right) 10\right|^{2}+\lambda^{2}|X|^{2}\left(\left|\phi^{+}\right|^{2}+\left|\phi^{-}\right|^{2}\right) \\
& -m^{2}\left(\left|\phi^{+}\right|^{2}+\left|\phi^{-}\right|^{2}\right)+\frac{1}{2} D_{1}^{2}+\frac{1}{2} D_{10}^{2}
\end{aligned}
$$

where $D_{1}=g_{1}\left(\left|\phi^{+}\right|^{2}-\left|\phi^{-}\right|^{2}+\cdots\right)$ and $D_{10}=g_{10}\left(|10|^{2}+\cdots\right)$ and the ellipsis refers to the contribution to $D_{1}\left(D_{10}\right)$ of all other $\mathrm{U}(1)(\mathrm{SO}(10))$ multiplets necessary to make this a complete theory.

A minimum is obtained for vacuum expectation values [vev] $<\phi^{+}>=$ $-<\phi^{-}>\equiv \phi,<X>\equiv X$ and $<10>=0$ with

$$
\phi^{2}=\frac{3 \lambda^{\prime} m^{2}}{\lambda^{3}} \frac{1}{\left(2-\lambda / 3 \lambda^{\prime}\right)}, \quad X^{2}=\frac{m^{2}}{\lambda^{2}}\left(\frac{1-\lambda / 3 \lambda^{\prime}}{2-\lambda / 3 \lambda^{\prime}}\right)
$$

and $\lambda / 3 \lambda^{\prime}<1$. The vev $<\mathcal{S}>\equiv M$ is undetermined in the global SUSY limit, at tree level.

At the minimum, SUSY is broken with

$$
F_{X}=\frac{m^{2}}{\lambda} \frac{1}{\left(2-\lambda / 3 \lambda^{\prime}\right)}
$$

and

$$
V_{\text {tree }}=F_{X}^{2}\left(1-6 \lambda^{\prime} / \lambda\right)<0 .
$$

${ }^{2}$ This model is not "natural" in the sense that it does not include all interactions not forbidden by symmetries. It is however "SUSY natural," since no new terms in the SUSY potential can be generated due to the non-renormalization theorems of SUSY. However an example of a "natural" theory of this type is given in Appendix 2. 
Note, $\sqrt{F_{X}} \sim X \sim m$; a property of the minimal model.

We now argue that $M$ is the messenger scale when $M>>m$ and in section 2.2 we show how the condition $M>>m$ can be obtained without any finetuning. The 10 is the only field which couples to $\mathrm{SO}(10)$ and feels SUSY breaking directly through $F_{X}$. The mass of the 10 is given by $\lambda_{\mathcal{S}} M+X \approx$ $\operatorname{Max}(M, m)$. Note, if $M \approx m$ then we have the light messenger scenario. If on the other hand $M>>m$, then the effective supersymmetry breaking scale is given by $\Lambda=F_{X} / M \approx m^{2} / M \sim 10-100 \mathrm{TeV}$ with both $M$ and $m$ much greater than $\Lambda$.

For $M_{G U T}>M$, gauginos, squarks and sleptons receive mass radiatively, predominantly through SM gauge interactions[1], 4, 7]. Gauginos obtain mass at one loop given by

$$
m_{\lambda_{i}}=c_{i} \frac{\alpha_{i}(M)}{4 \pi} \Lambda \quad(\text { for } i=1,2,3)
$$

where $c_{1}=\frac{5}{3}, c_{2}=c_{3}=1$.

The scalar masses squared arise at two-loops

$$
\tilde{m}^{2}=2 \Lambda^{2}\left[\sum_{i=1}^{3} C_{i}\left(\frac{\alpha_{i}(M)}{4 \pi}\right)^{2}\right]
$$

where $C_{3}=\frac{4}{3}$ for color triplets and zero for singlets, $C_{2}=\frac{3}{4}$ for weak doublets and zero for singlets, and $C_{1}=\frac{5}{3}\left(\frac{Y}{2}\right)^{2}$, with the ordinary hypercharge $Y$ normalized as $Q=T_{3}+\frac{1}{2} Y$. In the limit $M<<M_{G U T}$, we have $\alpha_{3}(M)>>\alpha_{2}(M)>\alpha_{1}(M)$. Thus squark doublets and singlets are approximately degenerate, while right-handed sleptons are expected to be the lightest SUSY partners of SM fermions.

Now consider the case $M \sim M_{G U T}$. In this case all $\mathrm{SO}(10)$ gauge bosons contribute equally to gaugino, squark and slepton masses. For gauginos we obtain a common mass at one loop

$$
m_{\lambda}=\frac{\alpha_{G U T}}{4 \pi} \Lambda
$$

and for squarks and sleptons a positive mass squared at two loops

$$
\tilde{m}^{2}=2 \Lambda^{2}\left[C_{10}\left(\frac{\alpha_{G U T}}{4 \pi}\right)^{2}\right]
$$

with $C_{10}=\frac{45}{8}$ for $16 \mathrm{~s}$ of $\mathrm{SO}(10)$. Hence, in this case, squarks and sleptons are degenerate, thus satisfying the boundary conditions usually assumed for minimal supergravity mediated SUSY breaking or the CMSSM. 
Note that the gaugino masses are the same order as the squark and slepton masses.3] This is because $W$ [ eqn. 3] has an R symmetry in which all fields have $\mathrm{R}$ charge 1 and $W$ has $\mathrm{R}$ charge 3 . This $\mathrm{R}$ symmetry must be broken in order to generate a gaugino mass. However the $\mathrm{R}$ symmetry breaking scale, $M_{R}=M$, is the same as the messenger scale; hence gaugino masses are not suppressed in this model. In the next section we give an example where the $\mathrm{R}$ symmetry breaking scale is much smaller than the messenger scale.

\subsection{Supergravity effects}

What about supergravity effects? For the range of parameters satisfying $\sqrt{3} M_{\text {Planck }}>>4 \pi M / \alpha$, the dominant contribution to observable scalar masses is gauge mediated. In the case $F \sim F_{X}, M \sim M_{G U T} \approx 2 \times 10^{16} \mathrm{GeV}$, and $\alpha_{G U T}^{-1} \approx 24$, the contribution of supergravity mediated SUSY breaking is comparable to that of gauge mediated SUSY breaking. This supergravity contribution to squark and slepton masses could lead to observable FCNC processes, if it does not respect flavor symmetry. In addition both contributions receive radiative corrections from physics between $M_{G U T}$ and $M_{\text {Planck }}$ which could also induce large flavor violating effects [6]. However both of these flavor violating contributions can be suppressed by reducing $M$. For example, with $M \sim M_{G U T} / 10$, the supergravity contribution to flavor violating scalar masses $\delta m^{2} / m^{2}$ is reduced to a few percent. The flavor violating GUT corrections to the gauge mediated scalar masses are also reduced by a similar factor $\left(M / M_{G U T}\right)^{2}$ (see Appendix 1). Thus both contributions to the real part of $\delta m^{2} / m^{2}$ are reduced to a few percent which appears to be safe[10]. Finally, for $M \sim M_{G U T} / 10$, squarks and sleptons are almost degenerate and thus approximately satisfy the boundary conditions usually assumed for the CMSSM [3, 8].

\footnotetext{
${ }^{3}$ Thus two of the independent parameters of minimal supergravity, the scalar mass parameter $m_{0}$ and the gaugino mass $M_{1 / 2}$ of minimal supergravity, are related.

${ }^{4}$ Supergravity will induce small contributions to scalar masses given by $m_{0} \approx m_{3 / 2}=$ $F / \sqrt{3} M_{\text {Planck }}$. It should be stressed that $F_{X}$ is not necessarily the intrinsic supersymmetry breaking scale, $F$, since the gauge singlet field may not be coupled directly to the supersymmetry breaking sector. For example, in the model of Dine, Nelson, Nir and Shirman[ [4], $F \gg F_{X}$. However, it is also perfectly possible that $F \sim F_{X}$ [9]. While $F_{X}$ determines the superpartner masses, it is $F$ which determines the gravitino mass.

${ }^{5}$ If $\Lambda=F / M$ is fixed, then $m_{0}^{2}=\left(F / \sqrt{3} M_{\text {Planck }}\right)^{2}=\Lambda^{2}\left(M / \sqrt{3} M_{\text {Planck }}\right)^{2}$.
} 


\subsection{Why is $M>>m$ ?}

How do we obtain $M>>m$ ? The effective potential for $\mathcal{S}$ is flat at tree level in the global SUSY limit. It receives significant contributions both from supergravity and at one loop given by

$$
V(\mathcal{S})=V_{\text {tree }}+m_{\mathcal{S}}^{2}(\mu) \mathcal{S}^{2}+16 F_{X}^{2} \ln [(\mathcal{S}+X) / m]
$$

where $m_{\mathcal{S}}^{2}(\mu)$ is the supergravity generated soft SUSY breaking mass term evaluated at the scale $\mu=\mathcal{S} . m_{\mathcal{S}}^{2}(\mu), m_{10}^{2}(\mu), m_{\lambda_{i}}(\mu), \alpha_{i}(\mu), \lambda_{\mathcal{S}}(\mu)$, etc. satisfy coupled RG equations schematically given by

$$
\begin{array}{rlrl}
\frac{d m_{\mathcal{S}}^{2}}{d t} & = & \frac{\lambda_{\mathcal{S}}^{2}}{4 \pi}\left(20 m_{\mathcal{S}}^{2}+40 m_{10}^{2}+\cdots\right) & \\
\frac{d m_{10}^{2}}{d t} & = & \frac{\lambda_{\mathcal{S}}^{2}}{4 \pi}\left(4 m_{\mathcal{S}}^{2}+8 m_{10}^{2}+\cdots\right) & -4 \alpha_{i} C_{i} m_{\lambda_{i}}^{2} \\
\frac{d}{d t}\left(\frac{m_{\lambda_{i}}}{\alpha_{i}}\right) & = & 0
\end{array}
$$

where the ellipsis refers to A terms which may or may not be significant, $t \equiv \ln \left(\mu / M_{\text {Planck }}\right) / 2 \pi, C_{i}$ are defined after eqn. (8) and we have not written the other equations. Note that the $R G$ equations for $m_{10}$ have both gauge and Yukawa contributions whereas for $m_{\mathcal{S}}$ only Yukawa contributions appear. Moreover $m_{\lambda_{3}}$ and $\alpha_{3}$ grow as $\mu$ decreases. As a result we expect that $m_{10}$ will vary slowly with scale, while $m_{\mathcal{S}}$, on the other hand, will vary significantly. The Yukawa interactions tend to drive $m_{\mathcal{S}}^{2}$ to negative values. For example the solution to the much simplified equation

$$
\frac{d m_{\mathcal{S}}^{2}}{d t}=\frac{\lambda_{\mathcal{S}}^{2}}{4 \pi}\left(40 m_{10}^{2}\right)=\text { constant }
$$

is given by

$$
m_{\mathcal{S}}^{2}(t)=m_{\mathcal{S}}^{2}(0)+\frac{\lambda_{\mathcal{S}}^{2}}{8 \pi^{2}}\left(40 m_{10}^{2}\right) \ln \left(\mu / M_{\text {Planck }}\right)
$$

If we now let $m_{\mathcal{S}}^{2}(0)=m_{10}^{2}=m_{0}^{2}$, ๆ we find

$$
m_{\mathcal{S}}^{2}(t)=m_{0}^{2}\left(1+40 \frac{\lambda_{\mathcal{S}}^{2}}{8 \pi^{2}} \ln \left(\mu / M_{\text {Planck }}\right)\right)
$$

\footnotetext{
${ }^{6}$ We have only included the supergravity contribution to scalar masses in the effective theory.
} 
which goes through zero at $\mu=M_{\text {Planck }} \exp \left[\frac{-8 \pi^{2}}{40 \lambda_{S}^{2}}\right]$. Since this mass term gives the dominant contribution to the effective potential for $\mathcal{S}$, it is clear that the value of $M=<\mathcal{S}>\approx M_{\text {Planck }} \exp \left[\frac{-\pi^{2}}{5 \lambda_{S}^{2}}\right]$ can take on any value between $M_{Z}$ and $M_{\text {Planck }}$ without any fine-tuning.

\section{Light Gluinos}

In the above case the mass of the 10 (the messenger mass $M$ ) is $\mathrm{SO}(10)$ invariant. We now discuss a simple variation of the model in which the messenger mass breaks $\mathrm{SO}(10)$. Consider the same superpotential as in eqn. (3) with the addition of a term

$$
10 A 10_{H}
$$

where $A, 10_{H}$ are an additional adjoint and ten dimensional representation of $\mathrm{SO}(10)$. We presume that $A$ is also included in an $\mathrm{SO}(10)$ breaking sector of the theory and obtains a vev of order $M_{G U T}$ in the B-L direction. As a result the effective $10-10_{H}$ mass term takes the form

$$
\frac{1}{2}\left(\begin{array}{ll}
10 & 10_{H}
\end{array}\right)\left(\begin{array}{cc}
2 \mathcal{S} & A \\
A & 0
\end{array}\right)\left(\begin{array}{c}
10 \\
10_{H}
\end{array}\right)
$$

In this case the color triplets in $10-10_{H}$ obtain mass of order $M_{G U T}$, while the doublets in $10,10_{H}$ obtain mass of order $M, 0$ respectively [11. The massless doublets may be identified as the MSSM electroweak higgs doublets.

Now if $M_{G U T}>>M>m$ we must carefully identify both the messenger scale, and the R symmetry breaking scale, $M_{R}$; the SUSY breaking scale is still $m$. First consider the $\mathrm{R}$ symmetry breaking scale. The vev of $A$ appears to break $\mathrm{R}$ symmetry and in fact it does break the $\mathrm{R}$ symmetry defined earlier. However it also breaks a global $\mathrm{U}(1)$ symmetry in which $10,10_{H}, A, \mathcal{S}$ have charge $0,-1,1,0$ respectively. Thus there is an $\mathrm{R}$ symmetry with charges $1,2,0,1$ which remains conserved. This $\mathrm{R}$ symmetry is sufficient to keep gauginos massless. The vev $\langle\mathcal{S}\rangle=M$ on the other hand, breaks this $\mathrm{R}$ symmetry; hence $M_{R}=M$.

There are two effective messenger scales in this model given by $M_{G U T}$ and $M$. In the color non-singlet sector, the messenger scale is fixed by the vev $\left\langle A>=M_{G U T} \times(B-L)\right.$, while in the color singlet sector the messenger 
scale is determined by the vev $\langle\mathcal{S}\rangle=M$. Thus there are now two effective SUSY breaking scales, $\Lambda_{C}=F_{X} / M_{G U T}$ in the color non-singlet sector and $\Lambda=F_{X} / M$ for color singlets. Note $\Lambda>\Lambda_{C}$ for $M<M_{G U T}$. We take $\Lambda \sim 10-100 \mathrm{TeV}$.

Integrating out the messenger sector in this case gives rise to gaugino masses at one loop. We find

$$
\begin{array}{lll}
m_{\lambda_{3}}= & c_{3} \frac{\alpha_{3}\left(M_{G U T}\right)}{4 \pi} \Lambda_{C}\left(\frac{M}{M_{G U T}}\right) \\
m_{\lambda_{i}}= & c_{i} \frac{\alpha_{i}(M)}{4 \pi} \Lambda \quad(\text { for } i=1,2) .
\end{array}
$$

Gluino masses are suppressed in this model by two factors of $\left(M / M_{G U T}\right)$. One factor comes from the ratio of the $\mathrm{R}$ symmetry breaking scale and the messenger scale for color interactions, and the other from the ratio of the two different effective SUSY breaking scales, $\Lambda_{C} / \Lambda=M / M_{G U T}$.

The scalar masses squared arise at two-loops

$$
\begin{array}{cc}
\tilde{m}_{\text {squark }}^{2}= & 2 \Lambda_{C}^{2}\left[C_{3}\left(\frac{\alpha_{3}\left(M_{G U T}\right)}{4 \pi}\right)^{2}\right] \\
& +2 \Lambda^{2}\left[C_{2}\left(\frac{\alpha_{2}(M)}{4 \pi}\right)^{2}+C_{1}\left(\frac{\alpha_{1}(M)}{4 \pi}\right)^{2}\right] \\
\tilde{m}_{\text {slepton }}^{2}=m_{\text {higgs }}^{2}= & 2 \Lambda^{2}\left[C_{2}\left(\frac{\alpha_{2}(M)}{4 \pi}\right)^{2}+C_{1}\left(\frac{\alpha_{1}(M)}{4 \pi}\right)^{2}\right] .
\end{array}
$$

For $\Lambda_{C} / \Lambda=M / M_{G U T}<<1$, electroweak doublet squarks and sleptons are approximately degenerate while right-handed squarks and sleptons have mass ratios determined by the square of their weak hypercharge.

\subsection{The gluino as the LSP}

The gluino-photino mass ratio, in our example, is given by

$$
\frac{m_{\tilde{g}}}{m_{\tilde{\gamma}}} \approx \frac{3 \alpha_{3}}{8 \alpha_{E M}}\left(\frac{M}{M_{G U T}}\right)^{2}
$$

The gluino is likely to be the lightest SUSY particle [LSP].7 If the gluino is light and long-lived it would have some interesting new signatures for

\footnotetext{
${ }^{7}$ The gluino-gravitino mass ratio, given by $\frac{\alpha_{3}\left(M_{Z}\right) \sqrt{3}}{4 \pi} \frac{M M_{P l a n c k}}{M_{G U T}^{2}} \frac{F_{X}}{F}$, is likely to be less than 1 .
} 
detectors [12, 13, 14, 15, 16]. Several authors have previously considered the possibility of gluinos being the LSP 17]. Assuming R parity is conserved the gluino would be absolutely stable. It would form color singlet bound states - a pseudo-scalar gluino-gluino composite $\eta_{\tilde{g}}$ (called a gluinoball) and a fermionic gluino-gluon composite $R_{0}$ [13, 14 (called a glueballino). For massless gluinos, the $\eta_{\tilde{g}}, R_{0}$ and the scalar $0^{++}$glueball form a massive supermultiplet with a dynamical mass determined by QCD. For non-zero gluino mass, we have $m_{\eta_{\tilde{g}}}>m_{R_{0}}>m_{\text {glueball }}$. In either case $\eta_{\tilde{g}}$ like the glueball is unstable, decaying predominantly into multi-pion final states, while $R_{0}$ may remain stable. These are just a couple of states in the large menagerie of R-hadrons [18, 19, 20]. There are also R-baryons (gluebarinos) and R-mesons (gluemesinos). Of the R-baryons, the flavor singlet $S_{0}=\tilde{g} u d s$ is expected to be the lightest[19]. It may also be long-lived or possibly even stable if $m_{S_{0}}<m_{R_{0}}+m_{n}$. Of the R-mesons, the isotriplet $\tilde{\rho}$, including the charged $\tilde{\rho}^{+}=\tilde{g} u \bar{d}$ and neutral $\tilde{\rho}^{0}=\tilde{g}(u \bar{u}-d \bar{d})$, is expected to be the lightest 20. It may be short-lived, decaying via strong interactions to $R_{0}+\pi$, or long-lived, decaying only via weak interactions to $R_{0}+\mu^{+}+\nu_{\mu}$ or $R_{0}+e^{+}+\nu_{e}$. It could even be stable, if it were the LSP. The glueballino $\left(R_{0}\right)$ and the R-rho $\left(\tilde{\rho}^{0}\right)$ are candidates for the LSP. We do not know apriori which of these is the lightest. For gluino masses greater than $3 \mathrm{GeV}$, bag model calculations suggest that $m_{\tilde{\rho}} \leq m_{R_{0}}$ [20]. However the ratio $m_{\tilde{\rho}} / m_{R_{0}}$ depends on a gluon self-energy parameter which is not well determined. Comparison of different bag model calculations of glueball and mixed glue- $q \bar{q}$ states leads us to expect uncertainties on the order of $100-200 \mathrm{MeV}$ [21, 22]. We shall thus consider two alternatives - either $R_{0}$ or $\tilde{\rho}^{0}$ as the LSP. We shall sometimes refer to the candidate R-hadron LSP generically as $\tilde{R}$. We shall also assume that the $S_{0}$ is unstable and decays with a lifetime $\sim 10^{-10}$ seconds, of order the $\Lambda^{0}$ baryon lifetime.

The most dramatic change in SUSY phenomenology with a stable gluino LSP is that the missing energy signature for SUSY is now gone. Recall that general gluino searches in accelerator experiments have used the missing energy signature to identify the production and subsequent cascade decay of the gluino. A stable gluino, however, avoids all of these constraints. In our model, gluinos produced in high energy accelerator experiments will hadronize until the $\tilde{R}$ is formed. Since the $\tilde{R}$ is strongly interacting it will be stopped in the hadronic calorimeter and all its kinetic energy will be visible. As a result, most quoted gluino limits 13 now disappear. However, for very heavy $\tilde{R}_{\mathrm{s}}$ a 
missing energy signal re-emerges which can, in principle, be used to place an upper limit on the gluino mass.

Stable particle searches now provide the best limits on the $\tilde{R}$ mass. These are of two types -

- mass spectrometer searches for heavy isotopes of hydrogen or oxygen, and

- accelerator based fixed target or collider experiments looking for stable charged or neutral hadrons.

The mass spectrometer searches for heavy isotopes of hydrogen provide severe constraints for a stable gluino LSP.

There have been several mass spectrometer searches for heavy $(\mathrm{Z}=1)$ isotopes of hydrogen 23, 24, referred to as $X^{+}$. These experiments find no evidence for $X^{+}$at an abundance relative to hydrogen given by

$$
\frac{n_{X^{+}}}{n_{H^{+}}}>10^{-18}-10^{-22}
$$

for $m_{X^{+}}$in the range from $2-350 \mathrm{GeV} 23$ or

$$
\frac{n_{X^{+}}}{n_{H^{+}}}>2 \times 10^{-28}
$$

for $1000>m_{X^{+}}>12 \mathrm{GeV} 24$. In order for these results to be relevant for gluinos, however, we must interpret $X^{+}$as a bound state of $\tilde{R}$ (i.e. $R_{0}$ or $\tilde{\rho}^{0}$ ) with hydrogen, deuterium or other light element, i.e. $X^{+}=\tilde{R} H^{+}, \tilde{R} D^{+}$. Of course, this can only rule out a stable $\tilde{R}$ if two conditions are satisfied -

1. $\tilde{R}$ in fact binds to light elements, and

2. $\tilde{R}$ is present at the experimentally accessible abundances.

We shall argue later that the expected abundance of $\tilde{R}$ is such that these experiments should have seen something if $\tilde{R}$ binds to hydrogen. Thus let us consider the question of binding. The strong interactions of $R_{0}$ or $\tilde{\rho}^{0}$ with hydrogen differ. An $R_{0}$ has strong interactions but it is not expected to behave like a neutron. The dominant contribution to the binding of deuterium comes from pion exchange, while $R_{0}$, containing no valence quarks, is unlikely to couple significantly to pions, rhos, etc. The dominant exchange is likely to 
be a glueball with mass of order $1.5 \mathrm{GeV}$. Thus the interaction range is short, about $\frac{1}{10} \mathrm{fm}$, and considering that the deuterium binding energy is already quite small, it is unlikely that the $R_{0}$ would bind to light nuclei. Hence it would not be seen in experiments searching for heavy isotopes of Hydrogen. The $\tilde{\rho}^{0}$, on the other hand, probably does exchange pions and is thus more likely to bind to hydrogen. This may exclude the $\tilde{\rho}^{0}$ (assuming it is the LSP) with mass greater than $2 \mathrm{GeV}$. Clearly further studies are necessary to decide this important issue.

Consider the case of $\tilde{\rho}^{0}$ as the LSP. Then it is likely that the charged states will either be stable or very long-lived.

- If the charged states are stable then this case is already ruled out by several complimentary experiments. Searches for heavy isotopes of hydrogen limit the relative abundance

$$
n_{\tilde{\rho}^{+}} / n_{H^{+}}<10^{-18}
$$

for $m_{\tilde{\rho}^{+}}>2 G e V$ 23, 24]. In addition, accelerator experiments exclude a long-lived $\tilde{\rho}^{+}$with mass in the range $4<m_{\tilde{\rho}^{+}}<10 \mathrm{GeV}$ and lifetime $\tau_{\tilde{\rho}^{+}}>5 \times 10^{-8}$ sec. [25] or with $1.9<m_{\tilde{\rho}^{+}}<13.6 \mathrm{GeV}$ and $\tau_{\tilde{\rho}^{+}}>10^{-7}$ sec. [26]. Thus we conclude that the $\tilde{\rho}^{+}$cannot be stable (LSP or otherwise) $]^{9}$

- If the $\tilde{\rho}^{+}$is unstable, then its lifetime is expected to be of order the neutron lifetime $\sim 10^{3}$ sec., since the $\tilde{\rho}^{+}-\tilde{\rho}^{0}$ mass difference would only be a few $\mathrm{MeV}$ and the decay would be via the weak interaction process $\tilde{\rho}^{+} \rightarrow \tilde{\rho}^{0}+e^{+}+\nu_{e}$. In this case, a Fermilab collider experiment is significant [27]. It excludes $\tilde{\rho}^{+}$with mass greater than $50 \mathrm{GeV}$. Thus a long-lived, unstable $\tilde{\rho}^{+}$is only allowed in the mass range $13.6<$ $m_{\tilde{\rho}^{+}}<50 \mathrm{GeV}$. Moreover, since $\tilde{\rho}^{0}$ and $\tilde{\rho}^{+}$are nearly degenerate, we have $13.6<m_{\tilde{\rho}^{0}}<50 \mathrm{GeV}$.

Note, if $\tilde{\rho}^{0}$ binds to hydrogen, then the mass spectrometer limit combined with the above accelerator data[25, 26] excludes the case $\tilde{\rho}^{0}$ as the LSP.

\footnotetext{
${ }^{8}$ Isospin symmetry, however, forbids the direct $\tilde{\rho}^{0} \tilde{\rho}^{0} \pi^{0}$ coupling.

${ }^{9} \mathrm{We}$ believe that it is extremely unlikely for a stable $\tilde{\rho}$ to survive in the narrow window between $1.5 \mathrm{GeV}$, associated with the expected dynamical mass due to QCD, and $1.9 \mathrm{GeV}$ from accelerator and mass spectrometer limits.
} 
Let us next consider the possibility that the glueballino, $R_{0}$, is the LSP and that the lightest charged or neutral gluebarino or gluemesino decays into $R_{0}$ with lifetime shorter than $\sim 10^{-8}$ seconds. What are the experimental limits on such a stable glueballino? The mass range between 2 and $4.2 \mathrm{GeV}$ is presently excluded for $m_{R_{0}}$ [12, 13, 14]. This follows from the absence of a peak in the photon energy spectrum in the process $\Upsilon \rightarrow \gamma \eta_{\tilde{g}}$ by CUSB 28 or in the process $\Upsilon^{\prime} \rightarrow \gamma \chi_{b}\left(1^{3} P_{1}\right)$ followed by $\chi_{b}\left(1^{3} P_{1}\right) \rightarrow g \tilde{g} \tilde{g}$ by Argus 29] or from a search for new hadrons with lifetimes greater than $10^{-7} \mathrm{sec}$, using time-of-flight in a 590m long neutral beam at FNAL by Gustafson et al. 30, 12]. Recently, the so-called light gluino window has been severely constrained by the analysis of $e^{+} e^{-} \rightarrow 4$ jets using LEP data [31, 32]. The present limit set by Aleph [32] is $m_{\tilde{g}}>6.3 \mathrm{GeV}$. Note, however that this bound is subject to theoretical uncertainties of higher order QCD effects on the 4 jet angular distributions 33]. In addition one should compare QCD with or without the effects of gluinos. This has not been done in this case. If we ignore, for the moment, the Aleph limit, then the light gluino window is still a viable option with only the mass range $2<m_{R_{0}}<4.2 \mathrm{GeV}$ excluded. If on the other hand, we accept the Aleph bound, then the mass range $m_{R_{0}}>6.3 \mathrm{GeV}$ is still allowed. Of course, this assumes that $R_{0}$ does not bind to hydrogen.

We now argue that if $\tilde{R}$ binds to hydrogen, it is excluded with mass $m_{\tilde{R}}>$ $2 \mathrm{GeV}$ by mass spectrometer searches for heavy hydrogen [23, 24]. Given that $\tilde{R}$ binds to hydrogen forming an $X^{+}$, we must calculate the expected abundance $n_{X^{+}} / n_{H^{+}}$. The cosmological abundance of $\tilde{R}$ is expected to be of order 34, 35, 36,

$$
\frac{n_{\tilde{R}}}{n_{H}} \approx 10^{-10}\left(\frac{m_{\tilde{R}}}{1 G e V}\right) .
$$

$\tilde{R}$ s will then bind to nuclei; some will be processed into light elements beginning at nucleosynthesis and this process will accelerate to heavier elements during stellar evolution. Those contained in heavy elements are likely to remain there today, since they are protected from annihilation by the coulomb barrier of the host nucleus. Those contained in light elements can undergo further annihilations in stellar interiors at sufficiently high temperatures (of order $10^{10}{ }^{\circ} \mathrm{K}$ corresponding to an $\mathrm{MeV}$ mean thermal energy). However the $X^{+}$s which have not been reprocessed through stars will remain today. The fraction of premordial hydrogen on earth (that which has not been processed through stars) is a number of order 1 [37]; hence the terrestrial abundance 
of $X^{+} \mathrm{s}$ is not expected to be significantly different than the cosmological abundance of $\tilde{R}$ s. As a consequence, $\tilde{R}_{\mathrm{s}}$ with mass greater than $2 \mathrm{GeV}$ and which bind to hydrogen are excluded. If the light gluino window has been closed by Aleph and $\tilde{R}$ binds to hydrogen, we must conclude that the gluino LSP scenario is excluded.

Even if $\tilde{R}$ does not bind to hydrogen, it is still likely to bind to heavy nuclei. In this context, a search for heavy isotopes of oxygen is significant [38]. No evidence was found for such a heavy isotope (HO) with an abundance $n_{H O} / n_{O}>$ few $\times 10^{-19}-10^{-16}$ for $20<m_{H O}<54$ amu corresponding to $4<m_{R_{0}}<38 \mathrm{GeV}$. Whether or not this constrains the theory however requires a detailed calculation of the processing of $\tilde{R}$ into heavy elements. A lower bound on the number of $\tilde{R}_{\mathrm{s}}$ on earth can be obtained by considering the flux of gluinos incident on the earth due to their production by cosmic rays in the upper atmosphere [39]. A crude estimate of this effect, assuming an incident flux of gluinos, $10^{-6}-10^{-8} \mathrm{~cm}^{-2} s^{-1}$, $\mathrm{T}$ incident on a $4 \mathrm{~km}$ column depth of water during the $10^{9}$ year age of the earth, gives an abundance $n_{\tilde{R}} / n_{H} \sim 10^{-18}-10^{-20}$. Clearly a more careful calculation is needed.

To summarize, if gluinos are the LSP then they will form stable hadrons. A stable charged R-hadron is ruled out by accelerator and mass spectrometer experiments. A neutral $R_{0}$ or $\tilde{\rho}^{0}$, is however not necessarily ruled out. For the glueballino $\left(R_{0}\right)$, we assumed that the higher mass, unstable charged states would have short enough lifetimes, $\leq 10^{-8}$ s, to escape detection. For the $\tilde{\rho}^{0}$, on the other hand, we assumed that the $\tilde{\rho}^{+}$is sufficiently long-lived that it would not have escaped detection in accelerator experiments looking for stable charged hadrons. If either binds to hydrogen, forming a stable heavy isotope $X^{+}$, then the combination of accelerator experiments and mass spectrometer searches excludes it with any mass. If they do not bind to hydrogen, then the best limit on the glueballino mass, $m_{R_{0}}>6.3 \mathrm{GeV}$, comes from Aleph 32 四 and on the $\tilde{\rho}^{0}$ mass, $50>m_{\tilde{\rho}^{0}}>13.6 \mathrm{GeV}$, comes from accelerator experiments [25, 26, 27]. Searches for anomalously heavy isotopes of heavy elements may constrain this mass range, but detailed calculations of the present abundance of such isotopes are needed.

\footnotetext{
${ }^{10}$ This flux is obtained using the known flux of cosmic ray protons and the data on charm production to estimate the gluino production rate 39 .

${ }^{11}$ If the higher order QCD corrections to the 4 jet angular correlations significantly affect the Aleph results then this constraint may disappear. In this case the light gluino window remains open.
} 
Finally, let us briefly consider the new signatures for SUSY at high energy accelerators. Squark decay now proceeds via $\tilde{q} \rightarrow q+\tilde{g}$, so that squark-antisquark production at an $e^{+} e^{-}$collider results in 4 jets in the final state[40]. Such events have been observed by the Aleph collaboration at LEP II [41]. Photinos would decay into 3 jets containing a quark-anti-quark pair and a gluino. Thus, slepton-anti-slepton production at an $e^{+} e^{-}$collider would have 2 leptons and up to 6 jets in the final state.

At a $p p$ or $\bar{p} p$ collider, gluino production will be copious. An energetic gluino is expected to produce an hadronic jet containing a single $\tilde{R}$. The $\tilde{R}$ will most likely be stopped in the detector; consequently all "kinetic" energy is visible in the detector. This statement has two significant consequences. Firstly, since only the kinetic energy $\left(E-m_{\tilde{R}}\right)$ is visible in the calorimeter, a very heavy $\tilde{R}$ would still have a missing energy signal. This effect could be used to bound the gluino mass from above. In addition, in the case that $\tilde{R}=R_{0}$ one must take into account that the cross section for $R_{0}$ nucleon scattering is expected to be only of order $40\left(m_{\pi} / m_{\text {glueball }}\right)^{2} \mathrm{mb}$, since it is predominantly due to glueball exchange in the t-channel. Thus it is suppressed compared to the typical nucleon-nucleon total cross-section. This effect must be taken into account when modeling $\frac{d E}{d x}$ for $R_{0}$ in a detector.

As a final note, it has been suggested that a stable glueballino might produce the observed anomallous muon showers from the X-ray binary star Cygnus X-3 42]. Although this possibility has been questioned by several authors [39, 43], it is not excluded for $m_{\tilde{R}} \leq 10 \mathrm{GeV}$.

\section{Conclusion}

We have discussed gauge-mediated SUSY breaking at intermediate scales. In this case the effective SUSY breaking scale $\Lambda=F_{X} / M \sim 10-100 \mathrm{TeV}$ but both the messenger scale $M$ and the SUSY breaking scale $\sqrt{F_{X}}$ are much greater than $\Lambda$.

In the case $M \sim M_{G U T} / 10$ and invariant under the grand unified gauge symmetry, we have boundary conditions (at $M$ ) for gauginos, squarks and sleptons which match those of minimal supergravity (or CMSSM) SUSY breaking schemes with $m_{0}$ and $M_{1 / 2}$ related. The advantage of the gauge mediated scheme is that the problem of flavor changing neutral currents can be mitigated or completely eliminated, depending on the messenger scale. 
In a particular example, we showed that the gluino can naturally be the LSP with very interesting consequences for experiment. In this example, there are two effective gauge-mediated SUSY breaking scales $\Lambda=\frac{F_{X}}{M} \sim$ $10-100 \mathrm{TeV}$ for color singlet states and $\Lambda_{C}=\frac{F_{X}}{M_{G U T}}$ for color non-singlet states.

The stable gluino window may be quite large. The crucial question is whether the LSP binds to hydrogen. If it does, then the window is closed by mass spectrometer searches for heavy isotopes of hydrogen. If it does not, then there are two possibilities - a $\tilde{\rho}^{0} \mathrm{LSP}$ is allowed for $50>m_{\tilde{\rho}^{0}}>$ $13.6 \mathrm{GeV}$, while a glueballino LSP is allowed for $m_{R_{0}} \geq 6.3 \mathrm{GeV}$. In either case, accelerator experiments looking for missing energy would not have seen the LSP, since the LSP is stopped in the hadronic calorimeter. Note, the high mass gluino limit could in principle be constrained by missing energy, since the rest mass would escape detection.

Finally, we did not discuss the $\mu$ problem in this paper. However, in our second example the messenger and Higgs sectors mix and there does not appear to be a symmetry which prevents the $\mu$ term from being generated radiatively (once SUSY and $\mathrm{SO}(10)$ breaking is included). However a more detailed model which includes the $\mathrm{SO}(10)$ breaking sector would be needed before this question can be addressed further.

\section{Appendix 1 - Soft SUSY breaking masses}

Soft SUSY breaking mass terms by definition preserve the property of SUSY to eliminate all quadratic divergences [5, 44]. However they have an additional property which concerns us here in the context of the flavor changing neutral current problem. Above the messenger scale these mass terms no longer scale as local dimension two (for scalars) or three (for gauginos) operators. In fact they scale as non-local operators; as a consequence the effective mass terms are momentum dependent at high energies. For example for scalars below the messenger scale we have

$$
\tilde{m}^{2} \phi^{*} \phi
$$

where the mass squared

$$
\tilde{m}^{2}=\frac{F_{X}^{2}}{M^{2}}
$$


is a constant. However for external momentum $p>>M$ the mass squared is momentum dependent

$$
m^{2}(p) \sim \frac{F_{X}^{2}}{p^{2}}
$$

So in general we have

$$
m^{2}(p) \sim \frac{F_{X}^{2}}{M^{2}+p^{2}} .
$$

Similarly, for gauginos we have

$$
m_{\lambda_{i}}(p) \sim \frac{F_{X} M_{R}}{p^{2}}
$$

for $p>>M . M_{R}$ is the $\mathrm{R}$ symmetry breaking scale.

How does this affect the FCNC problem? In the effective theory below $M$, soft SUSY breaking scalar masses $\left(\tilde{m}^{2}\right)$ are degenerate in a given charge sector. Thus flavor changing neutral current effects are suppressed. However GUT physics can induce threshold corrections which violate flavor symmetries [6]. If however $M<<M_{G U T}$ these corrections are suppressed and we have

$$
m_{\text {scalar }}^{2}=\tilde{m}^{2}\left(1+O\left(\left(\frac{M}{M_{G U T}}\right)^{2}\right)\right) .
$$

\section{Appendix 2 - A Natural Theory}

Consider the O'Raifeartaigh type theory with three $\mathrm{SO}(10)$ singlets, $X_{i}, i=$ $1,2,3$ with arbitrary couplings to the 10 and to two additional gauge singlets $P^{+}, P^{-}$. We have the superspace potential

$$
W=\left(\lambda_{1 i} X_{i}\right) M^{2}+\left(\lambda_{2 i} X_{i}\right) P^{+} P^{-}+\left(\lambda_{3 i} X_{i}\right) 10^{2}
$$

where $i=1,2,3$ is summed and $\lambda_{n i}, n=1,2,3$ are 9 arbitrary Yukawa couplings. It is then always possible to define 3 new gauge singlet fields $X, Y, Z$ in terms of $X_{i}$, so that the superspace potential has the form

$$
W=X M^{2}+\left(a_{1} X+b_{1} Y\right) P^{+} P^{-}+\left(a_{2} X+b_{2} Y+Z\right) 10^{2} .
$$

This theory has no supersymmetric ground state since

$$
\begin{array}{rc}
-F_{X}^{*}= & a_{1} P^{+} P^{-}+a_{2} 10^{2}+M^{2} \\
-F_{Y}^{*}= & b_{1} P^{+} P^{-}+b_{2} 10^{2} \\
-F_{Z}^{*}= & 10^{2}
\end{array}
$$


Consider the local minimum

$$
\begin{aligned}
<10> & =0, \quad<P^{+}>=-<P^{-}>=\rho, \\
a_{1}<X>+b_{1}<Y>=0 &
\end{aligned}
$$

with

$$
z \equiv<Z>\quad \text { and } \quad y \equiv\left(-a_{1}<Y>+b_{1}<X>\right) /\left(a_{1}^{2}+b_{1}^{2}\right)^{1 / 2}
$$

arbitrary.

At the minimum we have

$$
\rho^{2}=\left(\frac{a_{1}}{a_{1}^{2}+b_{1}^{2}}\right) M^{2}
$$

with

$$
\begin{gathered}
<F_{Z}>=<F_{10}>=<F_{P^{ \pm}}>=0, \quad a_{1}<F_{X}>+b_{1}<F_{Y}>=0 \\
<F_{y}>\equiv\left(-a_{1}<F_{Y}>+b_{1}<F_{X}>\right) /\left(a_{1}^{2}+b_{1}^{2}\right)^{1 / 2}=b_{1} M^{2} /\left(a_{1}^{2}+b_{1}^{2}\right)^{1 / 2}
\end{gathered}
$$

Thus supersymmetry is broken. The vevs for $y, z$ will be determined once radiative corrections and supergravity effects are included. The linear combination

$$
z^{\prime} \equiv \frac{\left(a_{2} b_{1}-b_{2} a_{1}\right)}{\sqrt{a_{1}^{2}+b_{1}^{2}}} y+z
$$

appears in the effective superspace potential

$$
W_{\text {effective }}=z^{\prime} 10^{2}
$$

and thus is expected to get a large vev as discussed in the paper. The orthogonal linear combination does not appear in the superspace potential.

\section{Acknowledgments}

This work is partially supported by DOE contract DOE/ER/01545-708. I would like to thank R. Boyd, G. Farrar, H. Goldberg, M. Sokolov and G. Steigman for interesting discussions and suggestions. 


\section{References}

[1] S. Dimopoulos and S. Raby, Nucl. Phys. B192, 353 (1981); M. Dine, W. Fischler, and M. Srednicki, Nucl. Phys. B189, 575 (1981) ; M. Dine and W. Fischler, Phys. Lett. B110, 227 (1982); M. Dine and M. Srednicki, Nucl. Phys. B202, 238 (1982); L. Alvarez-Gaumé, M. Claudson, and M. Wise, Nucl. Phys. B207, 96 (1982); C. Nappi and B. Ovrut, Phys. Lett. B113, 175 (1982).

[2] M. Dine and W. Fischler, Nucl. Phys. B204, 346 (1982); S. Dimopoulos and S. Raby, Nucl. Phys. B219, 479 (1983); J. Polchinski and S. Susskind, Phys. Rev. D26, 3661 (1982); T. Banks and V. Kaplunovsky, Nucl. Phys. B211, 529 (1983).

[3] A.H. Chamseddine, R. Arnowitt and P. Nath, Phys. Rev. Lett. 29, 970 (1982); R. Barbieri, S. Ferrara and C.A. Savoy, Phys. Lett. B119, 343 (1983); L.J. Hall, J. Lykken and S. Weinberg, Phys. Rev. D22, 2359 (1983); P. Nath, R. Arnowitt and A.H. Chamseddine, Nucl. Phys. B322, 121 (1983).

[4] M. Dine, A.E. Nelson and Y. Shirman, Phys. Rev. D51, 1362 (1995); M. Dine, A.E. Nelson, Y. Nir and Y. Shirman, Phys. Rev. D53, 2658 (1996).

[5] S. Dimopoulos and H. Georgi, Nucl. Phys. B193, 150 (1981); N. Sakai, Zeit. Phys. C11, 153 (1981).

[6] L.J. Hall, V.A. Kostelecky and S. Raby, Nucl. Phys. B267, 415 (1986); H. Georgi, Phys. Lett. 169B, 231 (1986). For more recent discussions, see R. Barbieri, L. Hall, A. Strumia, Nucl. Phys. B445, 219 (1995), Nucl. Phys. B449, 437 (1995); S. Dimopoulos and D. Sutter, Nucl. Phys. B452, 496 (1995); S. Dimopoulos, G. F. Giudice and N. Tetradis, Nucl. Phys. B454, 59 (1995); R. Barbieri, A. Romanino and A. Strumia, Phys. Lett. B369, 283 (1996).

[7] M. Dine, S. Dimopoulos, S. Raby, and S. Thomas, Phys. Rev. Lett. 76 , 3484 (1996); D. Stump, M. Weist, and C. P. Yuan, Phys. Rev. D54, 1936 (1996); S. Dimopoulos, S. Thomas, and J. D. Wells, Phys. Rev. D54, 
3283 (1996) and hep-ph/9609434; S. Dimopoulos and G.F. Giudice, hepph/9609344; S. Ambrosanio, G. Kane, G. Kribs, and S.P. Martin, Phys. Rev. D54, 5395 (1996); S.P. Martin, hep-ph/9608224 (1996). K.S. Babu, C. Kolda and F. Wilczek, Phys. Rev. Lett. 77, 3070 (1996); J. A. Bagger, K.T. Matchev, D.M. Pierce, and R.-J. Zhang, hep-ph/9611229.

[8] G.L. Kane, C. Kolda, L. Roszkowski and J.D. Wells, Phys. Rev. D49, 6173 (1994).

[9] K. Intriligator and S. Thomas, SLAC-PUB-95-7041.

[10] For a recent analysis, see F. Gabbiani, E. Gabrielli, A. Masiero, and L. Silvestrini, Nucl. Phys. B477, 321 (1996).

[11] S. Dimopoulos and F. Wilczek, Proceedings Erice Summer School, Ed. A. Zichichi (1981). For a recent discussion, see K.S. Babu and S.M. Barr, Delaware preprint BA-94-04 (1994).

[12] S. Dawson, E. Eichten and C. Quigg, Phys. Rev. D31, 1581 (1985).

[13] H.E. Haber, U.C. Santa Cruz preprint SCIPP 93/21, Invited talk presented at the SUSY-93 Workshop, Northeastern University, Boston, MA, 29 March-1 April 1993 (1993).

[14] G.R. Farrar, Phys. Rev. D51, 3904 (1995); and RU-95-17 (hepph/9504295) (1995).

[15] J.L. Hewett, T.G. Rizzo and M.A. Doncheski, SLAC preprint SLACPUB-7372, hep-ph/9612377 (1996).

[16] There are many papers on light gluinos. For example, see G. Farrar and P. Fayet, Phys. Lett. 76B, 575 (1978); R. Barbieri, L. Girardello and A. Masiero, Phys. Lett. 127B, 429 (1983); L. Clavelli, Phys. Rev. D46, 2112 (1992); M. Jezabek and J.H. Kühn, Phys. Lett. B301, 121 (1993); J. Ellis, D.V. Nanopoulos and D.A. Ross, Phys. Lett. B305, 375 (1993); G.R. Farrar, RU-94-35 (hep-ph/9407401) (1994); G.R. Farrar and A. Masiero, RU-94-38 (hep-ph/9410401) (1994); L. Clavelli and P.W. Coulter, Phys. Rev. D51, 1117 (1995); L. Roszkowski and M. Shifman, Phys. Rev. D53, 404 (1996). 
[17] G.R. Farrar, Phys. Rev. Lett. 53, 1029 (1984); J. Ellis et al., Nucl. Phys. B238, 453 (1984); R. Barbieri and L. Maiani, Nucl. Phys. B243, 429 (1984).

[18] G.R. Farrar and P. Fayet, Phys. Lett. 76B, 442, 575 (1978).

[19] F. Buccella, G.R. Farrar and A. Pugliese, Phys. Lett. 153B, 311 (1985).

[20] M. Chanowitz and S. Sharpe, Phys. Lett. 126B, 225 (1983).

[21] see for example, M. Chanowitz and S. Sharpe, Nucl. Phys. B222, 211 (1983); C.E. Carlson, T.H. Hansson and C. Peterson, Phys. Rev. D30, 1594 (1984).

[22] G.R. Farrar, RU-94-35 (hep-ph/9407401) (1994).

[23] R.A. Muller et al., Science(April 29, 1977), 521; P.F. Smith and J.R.J. Bennett, Nucl. Phys. B149, 525 (1979).

[24] P.F. Smith et al., Nucl. Phys. B206, 333 (1982).

[25] D. Cutts et al., Phys. Rev. Lett. 41, 363 (1978).

[26] R. Akers et al. (OPAL Collaboration), CERN-PPE/95-021 (1995).

[27] F. Abe et al., Phys. Rev. Lett. 63, 1447 (1989).

[28] P.M. Tuts et al., Phys. Lett. 186B, 233 (1987).

[29] Argus collaboration, Phys. Lett. 167B, 360 (1986).

[30] H.R. Gustafson et al., Phys. Rev. Lett. 37, 474 (1976).

[31] F. Csikor and Z. Fodor, CERN preprint CERN-TH/96-323 (1996); A. de Gouvea and H. Murayama, HEP preprint hep-ph/9606449 (1996).

[32] The ALEPH Collaboration, CERN preprint PPE/97-002, January 8, (1997).

[33] G.R. Farrar, Phys. Lett. B265, 395 (1991).

[34] Ya. B. Zel'dovich, L.B. Okun and S.B. Pikel'ner, Phys. Lett. 17164 (1965) and Sov. Phys. Usp. 8, 702 (1966). 
[35] G. Steigman, Cargese Lect. in Phys. 6, 505 (1973) and Ann. Rev. Astron. Astrophys. 14, 339 (1976); B.W. Lee and S. Weinberg, Phys. Rev. Lett. 39, 165 (1977); S. Wolfram, Phys. Lett. B82, 65 (1979).

[36] C.B. Dover, T.K. Gaisser and G. Steigman, Phys. Rev. Lett. 42, 1117 (1979).

[37] G. Steigman, private communication.

[38] R. Middleton et al., Phys. Rev. Lett. 43, 429 (1979).

[39] M.B. Voloshin and L.B. Okun, Sov. J. Nucl. Phys. 43, 495 (1986).

[40] G.R. Farrar, RU-95-82 (hep-ph/9512306) (1995).

[41] ALEPH Collaboration, D. Buskulic et al., CERN preprint PPE/96-052 (1996).

[42] G. Auriemma, L. Maiani and S. Petrarca, Phys. Lett. 164B, 179 (1985);

V.S. Berezinsky and B.L. Joffe, ITEP-127, Moscow (1985).

[43] V.S. Berezinsky, J. Ellis and B.L. Joffe, Phys. Lett. 172B, 423 (1986).

[44] L. Girardello and M.T. Grisaru, Nucl. Phys. B194, 65 (1982). 